\title{
Unusual intron in the second exon of a Type III polyketide synthase gene of Alpinia calcarata Rosc.
}

\author{
Edayileveettil K Radhakrishnan, Rintu T Varghese and Soniya E Vasudevan \\ Plant Molecular Biology Division, Rajiv Gandhi Centre for Biotechnology, Thiruvananthapuram, \\ Kerala, India.
}

\begin{abstract}
Plant phenolic compounds form a valuable resource of secondary metabolites having a broad spectrum of biological activities. Type III polyketide synthases play a key role in the formation of basic structural skeleton of the phenolic compounds. As a group of medicinal plants, PKSs with novel features are expected in the genome of Zingiberaceae. The genomic exploration of PKS in Alpinia calcarata conducted in this study identified the presence of an unusual intron at the region forming the second exon of typical PKSs, forming a gateway information of distribution of novel PKSs in Zingiberaceae.
\end{abstract}

Key words: Alpinia calcarata, CHS, Type III polyketide synthase, phylogenetic analysis.

Received: July 30, 2009; Accepted: November 11, 2009.

The Type III polyketide synthase (PKS) superfamily of enzymes play an important role in the biosynthesis of phenolic compounds of diverse structure and function in plants (Austin and Noel, 2003). Chalcone synthase is the most common and widely distributed member of the PKS superfamily, consisting of homodimers of a $40-45 \mathrm{kDa}$ polypeptide. In the typical enzymatic reaction, PKS forms the chalcone by stepwise decarboxylative condensation of one coumaroyl CoA molecule with three malonyl CoA moieties, followed by a Claisen type cyclization of the tetraketide product (Ferrer et al., 1999). The amazing biochemical diversity of PKSs is shown by the identification and characterization of expanding members of the family, such as the 2-pyrone synthase, stilbene synthase, benzalacetone synthase, valerophenone synthase, acridon synthase, etc. (Austin and Noel, 2003, Radhakrishnan et al., 2009). But considering the huge metabolic complexity of plants, more members are very likely to occur in plants, especially in medicinal plants.

Polyketide synthases have highly conserved stretches of amino acid residues forming the catalytic pockets, and the residues forming these pockets are located mainly within the second exon (Brand et al., 2006). This facilitates the use of PCR based methods to identify the genomic distribution of PKSs in taxonomically diverse plants. The genomic information derived from such studies can give molecular insights into functional novelties, since the sub-

Send corrrespondence to E.V. Soniya. Plant Molecular Biology Division, Rajiv Gandhi Centre for Biotechnology, 695014 Thiruvananthapuram, Kerala, India. E-mail: evsoniya@rgcb.res.in. stitution of key amino acid residues may have a tremendous impact on the PKS reaction mechanism. Zingiberaceae plants are well known for their metabolite richness, and the distribution of structurally diverse phenolic compounds across the species reflects the genomic possibly novel PKS forms. So it is very probable that PKSs in Zingiberaceae might have been subject to remarkable genomic changes. In the current study, a PCR based investigation of a PKS gene was conducted in Alpinia calcarata, to unveil its genomic features.

Plants of Alpinia calcarata Rosc. were obtained from the Kerala Ayurvedic Research Institute, Thiruvananthapuram, Kerala, and were grown and maintained in the experimental plant garden at Rajiv Gandhi Centre for Biotechnology, Thiruvananthapuram, Kerala. Genomic DNA was isolated from young leaves by a modified CTAB protocol (Murray and Thompson, 1980). The quality of the DNA was analyzed by agarose gel electrophoresis and was quantified spectrophotometrically using Biospec-1601, DNA/Protein/Enzymes Analyzer (Shimadzu).

Primers for amplifying the second exon of PKS of $A$. calcarata were designed manually. For this, the PKS gene sequences reported from other plants were retrieved from NCBI and were aligned using the Clustal W program (Thompson et al., 1994). Sequences at the most conserved region were selected for designing the primers GPKSF ( $5^{\text {' }}$ CCTCGCCAAGGACCTCGCCGAGAACAA-3') and GPKSR (5' CGGACCGAACCCGAAGAGAACGCCCC A-3'). The PCR reaction mix $(50 \mu \mathrm{L})$ contained $100 \mathrm{ng}$ of genomic DNA, 20 pmol of each primer, 1.5 units of Taq 
DNA polymerase (Promega), $1.5 \mathrm{mM} \mathrm{MgCl}_{2}$ and $200 \mu \mathrm{M}$ of each dNTPs. Amplifications were carried out in a BioRad iCycler using the following conditions: initial denaturation for $5 \mathrm{~min}$ at $94{ }^{\circ} \mathrm{C}, 35$ cycles of $94{ }^{\circ} \mathrm{C}$ for $30 \mathrm{~s}$, $65^{\circ} \mathrm{C}$ for $1 \mathrm{~min}$ and $72{ }^{\circ} \mathrm{C}$ for $1 \mathrm{~min}$, followed by a final extension at $72{ }^{\circ} \mathrm{C}$ for $7 \mathrm{~min}$. The PCR products were analyzed on $2 \%$ agarose gel and purified using the GFX gel band purification kit (Amersham). The purified PCR product was ligated into pGEM-T Easy plasmid vector (Promega). The ligation reaction mix $(10 \mu \mathrm{L})$ contained $1 \mathrm{X}$ ligation buffer, $50 \mathrm{ng}$ of PCR product, $50 \mathrm{ng}$ of pGEM-T Easy vector DNA and 3 units of T4 DNA ligase. The ligation was carried out overnight at $4{ }^{\circ} \mathrm{C}$ and the product was used for transformation. Positive transformants were selected by blue white screening and colony PCR. Plasmid isolation was carried out from positive colonies by alkaline lysis (Birnboim and Doly, 1979) and sequenced using the Big Dye Terminator Cycle Sequencing Ready Reaction Kit Version.3.1 (Applied Biosystem).

Following BLAST analyses (BLASTN and BLASTX), multiple sequence alignments with selected PKSs were run using the Clustal $\mathrm{W}$ program. For this the sequences were first aligned at the amino acid level and subsequently, the nucleic acid sequences were aligned according to the amino acid sequences. Intron prediction was done by using the intron prediction program at NetPlantGene Server.

Phylogenetic analysis was carried out using the Neighbour Joining (NJ) and Maximum Parsimony (MP) methods implemented in MEGA3 (Kumar et al.,2004). The intron sequence of AcPKS was removed manually and was used for the phylogenetic analysis along with other sequences. Tree topology robustness was assessed by bootstrap analysis with 1000 resampling replicates for the NJ and MP methods. The accession numbers of the PKS sequences selected for the phylogenetic analysis along with AcPKS are as follows; Aloe arborescens PCS (AY823626), Aloe arborescens OKS (AY567707), Arabis alpina CHS (AF112084), Arachis hypogaea RES (DQ124938), Arabidopsis thaliana CHS (NM_121396, Bromheadia finlaysoniana CHS 3 (AF007097), Callistephus chinensis CHS (Z67988), Camellia sinensis CHS (D26593), Cardamine amara CHS (AF112085), Daucus carota CHS (AJ006780), Dictamnus albus CHS (AJ850132), Escherichia coli fabH (M96793), Gerbera hybrida 2-PS (Z38097), Humulus lupulus VPS (AB047593), Hordeum vulgare CHS (X58339), Hydrangea macrophylla CTAS (AB011468), Hypericum androsaemum BPS (AF352395), Ipomoea purpurea CHS (AB001826), Ipomoea batatas CHS (AB037389), Lilium hybrid CHS (AF169798), Medicago sativa CHS (L02901), Oryza sativa CHS (X89859), Petunia hybrida
CHS (X14593), Phalaenopsis sp. 'pSPORT1' BBS (X79903), Phaseolus vulgaris CHS (X06411), Pinus strobus CHS (AJ004800), Pisum sativum CHS (X63335), Psilotum nudum STS (AB022685), Rheum palmatum BAS (AF326911), Rheum palmatum ALS (AY517486), Rorippa amphibia CHS (AF144530), Ruta graveolens ACS (AJ297786), Sorghum bicolor CHS (AY069951), Triticum aestivum CHS (AY286098), Vitis vinifera STS (EF192465), Wachendorfia thyrsiflora PKS (AY727928), Zingiber officinale PKS (DQ486012), Brassica napus CHS (AF076333), Dendrobium nobile CHS (ABE77392), Iris germanica CHS (BAE53636), Curcuma longa CURS3 (AB506763), Curcuma longa CURS2 (AB506762), Curcuma longa CURS1 (AB495007), Curcuma longa DCS(AB495006), Oryza sativa CUS (AK109558) and Streptomyces griseus RppA (AB018074).

Use of PKS specific primers for the PCR resulted in the formation of a $703 \mathrm{bp}$ product from genomic DNA of Alpinia calcarata (Figure 1). This amplicon size was higher than expected because the primers were selected to give a $600 \mathrm{bp}$ product. This was also expected from our previous experiments on the PKS gene family in Zingiber officinale. So the genomic DNA of $Z$. officinale was used as a control. In the BLAST analysis, the nucleotide sequence of $A$. calcarata PKS identified in this study (AcPKS, GenBank accession number EU399815) showed a highest identity of $87 \%$ to the PKS/CHS of Zingiber officinale, with the core fragment coinciding with the region between 546-1101 bp of typical PKS/CHS. Type III PKSs in plants generally have a size of $1170 \mathrm{bp}$, coding for 390 amino acids. The most characteristic feature of the core region is its location in the second exon, which contains most of the residues of the catalytic region. Comparative analysis of PKS/CHS sequences from all the plants revealed that all of them have only one intron, except for Antirrhinum majus (Sommer and Saedler, 1986). The first exon of CHS encodes 37-64 amino acid residues and the second exon codes for about 340 amino acid residues. Intron size can, however, vary from less than $100 \mathrm{bp}$ to several kilobases (Oberholzer et al., 2000). Due to the unusual size of AcPKS, it formed a gap of $93 \mathrm{bp}$ in the BLAST analysis, making the genomic data more interesting. This was also reflected in the multiple sequence alignment confirming that the extra sequence is specific to AcPKS and is very unusual. Intron prediction analysis identified a potential donor splice site at $217 \mathrm{bp}$ and an acceptor splice site at the $310 \mathrm{bp}$ position in AcPKS (Figure 1). The intron was found to have a size of $93 \mathrm{bp}$, and its presence at this region is very uncommon or even novel. The intron sequence was removed manually and the coding region was translated to amino acid sequences and compared with the CHS of $M$. sativa. The comparative analysis 


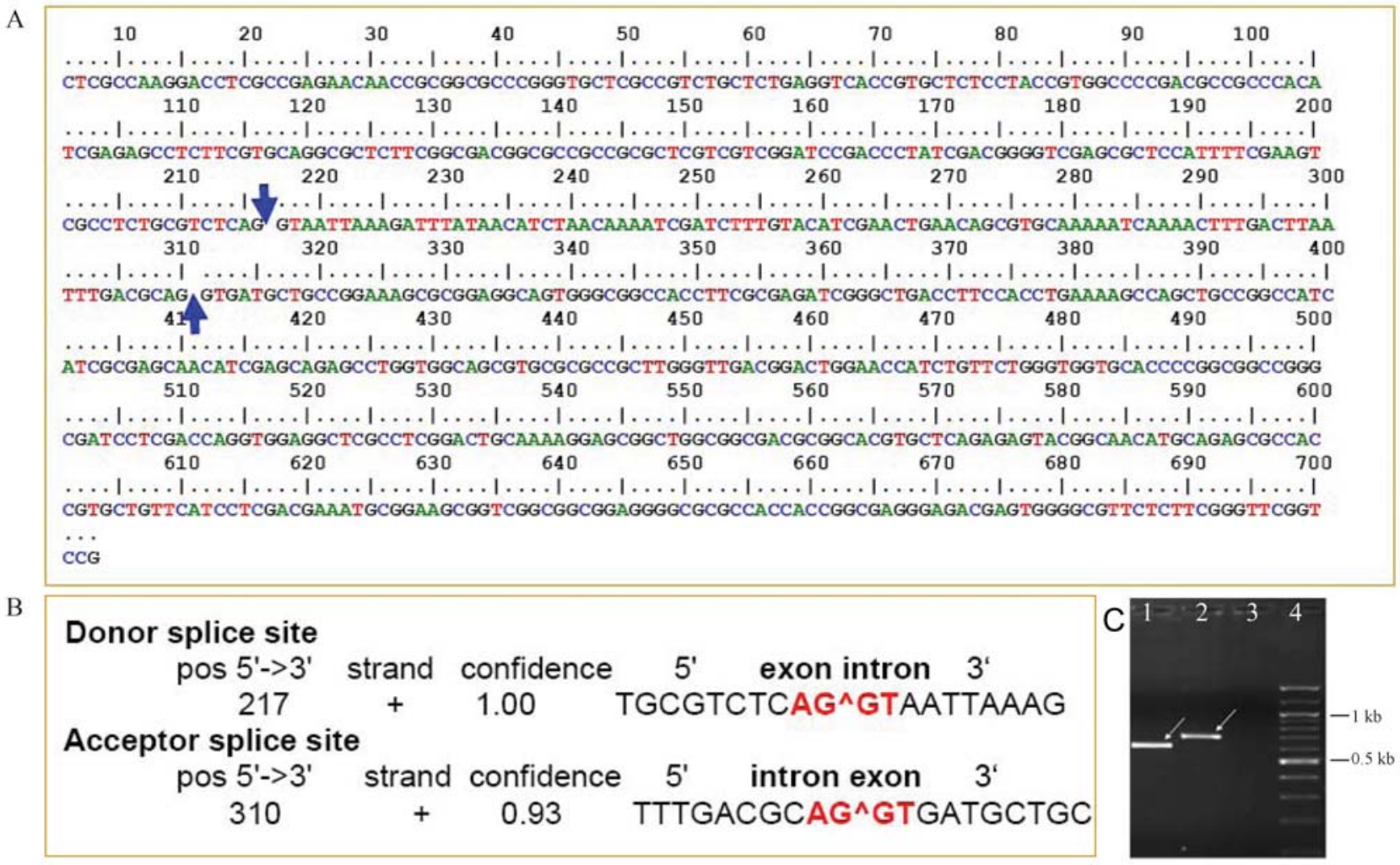

Figure 1 - A. Intron prediction for the Alpinia calcarata PKS sequence carried out at the NetPlantGene web server. The arrows represent putative splice sites. B. A donor splice site predicted at $217 \mathrm{bp}$ and an acceptor splice at the $310 \mathrm{bp}$ position. C. Amplification of a core fragment of the Alpinia calcarata PKS gene. Lanes (1) PCR product obtained from genomic DNA of Zingiber officinale, used as positive control, (2) PCR product obtained from genomic DNA of Alpinia calcarata, (3) negative control, and (4) 100 bp DNA ladder.

showed remarkable substitutions of amino acid residues in A. calcarata: Thr (197) to Ser, Ser (338) to Gln, Ileu (254) to Val, when compared to typical CHSs. The numbering and type of amino acid residues is based on the amino acid sequence of $M$. sativa CHS. These changes observed on $A$. calcarata $\mathrm{PKS}$ can be taken as an indication of function(s) other than typical chalcone formation. The presence of the unusual 93 bp intron in A. calcarata may be of significance to the evolution of the PKS gene family in Zingiberaceae. All CHS genes studied so far contained an intron at a conserved position, but in Antirrhinum majus a second intron was found to be present in the second exon (Sommer and Saedler, 1986). Very recently, CHS superfamily members with two introns or without an intron have been reported from Physcomitrella patens (Jiang et al., 2006). Yet, the current result is the first report of an intron in the second exon of PKS from Zingiberaceae. A recent report of a novel Type III polyketide synthase encoded by a three-intron gene in Polygonum cuspidatum (Ma et al., 2009) makes the current result even more interesting.

The phylogenetic analysis of AcPKS with other PKS sequences showed separate clustering, distinct from typical CHS/PKSs and along with recently identified plantspecific nonchalcone forming PKSs (Figure 2). The sepa- rate clustering of the sequence may be an indication of its biochemical distinctness. The clustering of AcPKS with the recently characterized PKSs of the curcuminoid synthase group also supports its this view (Katsuyama et al., 2007, Katsuyama et al., 2009a, and Katsuyama et al., 2009b). All the nonchalcone forming PKSs form a separate cluster, different from the typical CHSs. The biosynthetic role of nonchalcone forming PKSs is well studied and shown to play key role in building the structural skeleton of medicinal natural products, and some of them may even form unnatural compounds (Abe et al., 2002). In the case of Rheum palmatum, a phylogenetic analysis including four members of the PKS superfamily generated separate clusters for CHSs and non CHSs. Such separate clustering was also reflected in the biochemical activities (Abe et al., 2005).

Most of the genes coding for the biosynthetic machinery of plant secondary metabolism are encoded by small families of genes originated through duplication over evolutionary time (Durbin et al., 2000). Type III polyketide synthase or chalcone synthase occurs in most plants as multigene families (Radhakrishnan and Soniya, 2009). In legumes, it forms multigene family of 6 to 12 members (Tuteja et al., 2004; Matsumura et al., 2005). The genomic 


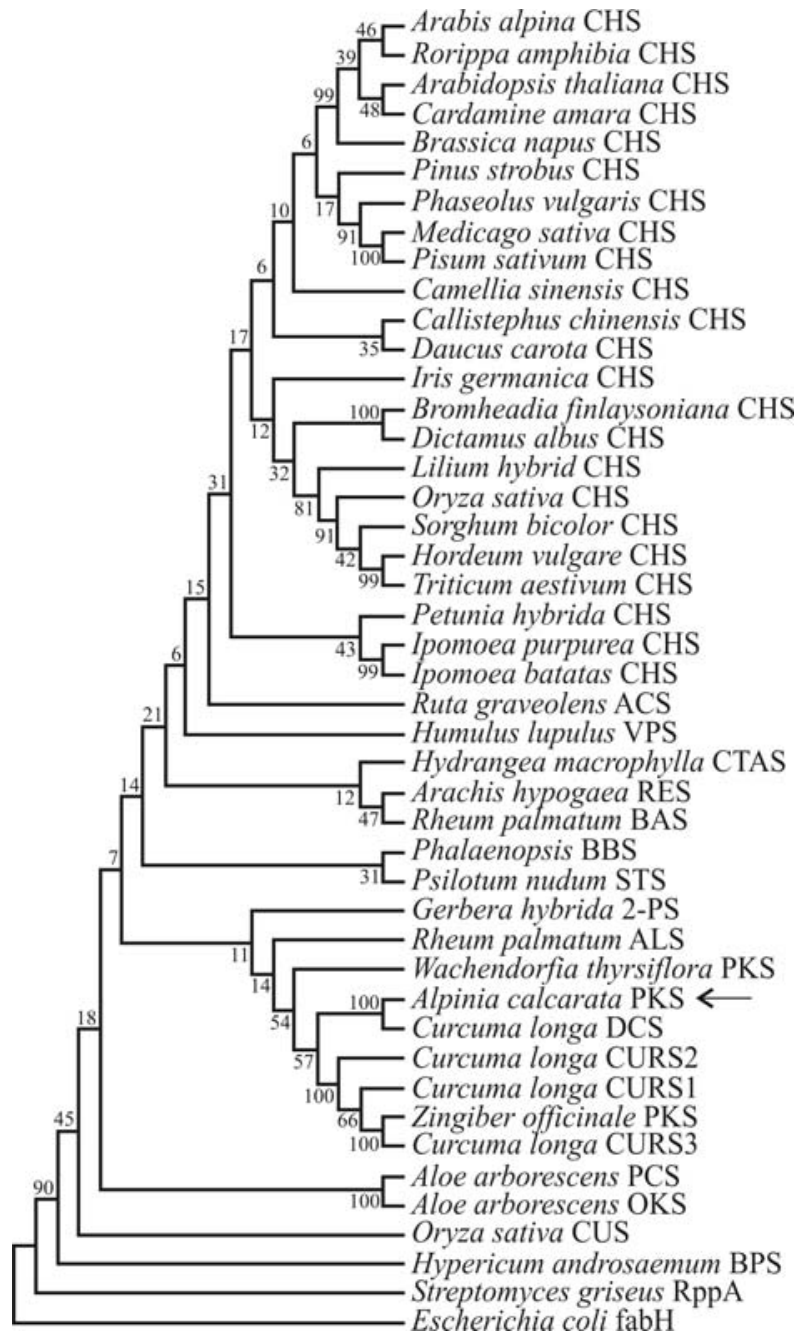

Figure 2 - Phylogenetic analysis of AcPKS with other members of PKS superfamily, using the NJ method implemented in MEGA 3 and 1000 bootstrap replicates. The AcPKS identified in this study is marked by an arrow. It forms a cluster with members of the nonchalcone forming PKSs. Abbreviations: CHS - chalcone synthase; STS - stilbene synthase; 2-PS 2-pyrone synthase; ACS - acridone synthase; ALS - aleosine synthase; BBS - bibenzyl synthase; CTAS - 4-coumaroyltriacetic acid synthase; BAS - benzalacetone synthase; VPS - valerophenone synthase; PKS polyketide synthase; RES - resveratrol synthase; BPS - benzophenone synthase; PCS - pentaketide chromane synthase; OKS - octaketide chromane synthase; RppA - red-brown pigment producing enzyme; FABH b-ketoacyl carrier protein synthase III (out group), CUS and CURS curcuminoid synthase; DCS - diketide CoA synthase.

distribution of the PKS superfamily in medicinal plants is yet little investigated and their analysis may result in novel insights into the functional and evolutionary features of PKSs. Sequence analysis of the second exon of $A$. calcarata PKS identified in this study shows the unusual occurrence of an intron that can be considered as a novelty. The sequence identified in this study opens a perspective for further molecular exploration of the PKS family in $A$. calcarata.

\section{Acknowledgments}

Radhakrishnan E.K. gratefully acknowledges a Senior Research Fellowship from the Council of Scientific and Industrial Research (CSIR), New Delhi, India.

\section{References}

Abe I, Takahashi Y and Noguchi H (2002) Enzymatic formation of an unnatural C6-C5 aromatic polyketide by plant type III polyketide synthases. Org Lett 4:3623-3626.

Abe I, Watanabe T and Noguchi H (2005) Chalcone synthase superfamily of type III polyketide synthases from rhubarb (Rheum palmatum). Proc Jpn Acad Ser B 81:434-440.

Austin MB and Noel JP (2003) The chalcone synthase superfamily of type III polyketide synthases. Nat Prod Rep 20:79-110.

Birnboim HC and Doly J (1979) A rapid alkaline extraction procedure for screening recombinant plasmid DNA. Nucleic Acids Res 7:1513-1523.

Brand S, Holscher D, Schierhorn A, Svatos A, Schroder J and Schneider B (2006) Type III polyketide synthase from Wachendorfia thyrsiflora and its role in diarylheptanoid and phenylphenalenone biosynthesis. Planta 224:413-428.

Durbin ML, McCaig B and Clegg MT (2000) Molecular evolution of the chalcone synthase multigene family in the morning glory genome. Plant Mol Biol 42:79-92.

Ferrer JL, Jez JM, Bowman ME, Dixon RA and Noel JP(1999) Structure of chalcone synthase and the molecular basis of plant polyketide biosynthesis. Nat Struct Biol 6:775-784.

Jiang C, Schommer CK, Kim SY and Suh DY(2006) Cloning and characterisation of chalcone synthase from the moss. Phytochemistry 67:2531-2540.

Katsuyama Y, Matsuzawa M, Funa N and Horinouchi S (2007) In vitro synthesis of curcuminoids by type III polyketide synthase from Oryza sativa. J Biol Chem 282:37702-37709.

Katsuyama Y, Kita T, Funa N and Horinouchi S (2009a) Curcuminoid biosynthesis by two type III polyketide synthases in the herb Curcuma longa. J Biol Chem 284:11160-11170.

Katsuyama Y, Kita T and Horinouchi S (2009b) Identification and characterization of multiple curcumin synthases from the herb Curcuma longa. FEBS Lett 583:2799-2803.

Kumar S, Tamura K and Nei M (2004) Integrated Software for Molecular Evolutionary Genetics Analysis and Sequence Alignment. Brief Bioinform 5:150-163.

Ma LQ, Pang XB, Shen HY, Pu GB, Wang HH, Lei CY, Wang H, Li GF, Liu BY and Ye HC (2009) A novel type III polyketide synthase encoded by a three- intron gene from Polygonum cuspidatum. Planta 9:457-469.

Matsumura H, Watanabe S, Harada K, Senda M, Akada S, Kawasaki S, Dubouzet EG, Minaka N and Takahashi R (2005) Molecular linkage mapping and phylogeny of the chalcone synthase multigene family in soybean. Theor Appl Genet 110:1203-1209.

Murray MG and Thompson WF(1980) Rapid isolation of high molecular weight plant DNA. Nucleic Acids Res 8:43214325.

Oberholzer V, Durbin ML and Clegg MT(2000) Comparative genomics of chalcone synthase and Myb genes in the grass family. Genes Genet Syst 75:1-16. 
Radhakrishnan EK and Soniya EV (2009) Molecular analysis of type III polyketide synthase (PKS) gene family from Zingiber officinale Rosc. Afr J Plant Sci 3:44-48.

Radhakrishnan EK, Sivakumar KC and Soniya EV (2009) Molecular characterization of novel form of Type III polyketide synthase from Zingiber Officinale Rosc. and its analysis using bioinformatics method. J Proteom Bioinform 2:310-315.

Sommer H and Saedler H (1986) Structure of the chalcone synthase gene of Antirrhinum majus. Mol Gen Genet 202:429-434.

Thompson JD, Higgins DG and Gibson TJ (1994) CLUSTALW: Improving the sensitivity of progressive multiple sequence alignment through sequence weighting, position specific gap penalties and weight matrix choice. Nucleic Acids Res 22:4673-4680.

Tuteja JH, Clough SJ, Chan WC and Vodkin LO(2004) Tissue-specific gene silencing mediated by a naturally occurring chalcone synthase gene cluster in Glycine max. Plant Cell 16:819-835.

\section{Internet Resources}

NetPlantGene $\quad$ Server: http://www.cbs.dtu.dk/services/NetPGene/.

Associate Editor: Márcio de Castro Silva Filho

License information: This is an open-access article distributed under the terms of the Creative Commons Attribution License, which permits unrestricted use, distribution, and reproduction in any medium, provided the original work is properly cited. 